\title{
Significados acerca de la atención educativa en el aula regular a niñas y niños en situación de discapacidad en tres instituciones educativas*
}

\section{Meanings about the educational services in the regular classroom for children with disabilities in three educational institutions}

\author{
Melba Ximena Figueroa Ángel ${ }^{* *}$ \\ Universidad Santo Tomás \\ Bogotá, Colombia
}

Recibido: 24 de marzo de 2011 Revisado: 29 de mayo de 2011 Aceptado: 30 de junio de 2011

\section{Resumen}

Esta investigación tuvo como objetivo comprender los significados acerca de la atención educativa en el aula regular a niñas y niños en situación de discapacidad, en tres instituciones educativas. Para ello, se planteó una investigación cualitativa que se desarrolló a partir de algunos principios de la etnometodología. La información se obtuvo a través de estrategias de investigación como la entrevista a profundidad, el cuento y la comprensión de documentos que involucraban a los que se consideran principales protagonistas de la atención educativa en el aula regular de niñas y niños en situación de discapacidad: niñas y niños, padres o acudientes, profesores y directivas de las instituciones. Esta información se sistematizó a través del análisis de contenido de tipo categorial, codificación y vaciado en matrices. Como producto del desarrollo de esta investigación se identificaron los significados desde tres subcategorías: canónico, excepcional y negociaciones con respecto a la discapacidad, la integración y la inclusión escolar.

Palabras clave: educación, discapacidad, integración, inclusión, significados, acciones.

** Correspondencia: Ximena Figueroa, docente investigadora, Facultad de Psicología Universidad Santo Tomás. Correo electrónico: melbafigueroa@usantotomas.edu.co. La investigación contó con la colaboración de Diana Marcela López y Adriana Díaz Chávez psicólogas en formación. 


\section{Abstract}

This research aimed at understanding the meanings about the educational services in the regular classroom for children with disabilities in three educational institutions. To do this, it proposes a qualitative research developed from some guidelines of the ethomethodology.

The information was obtained through research strategies such as participant observation, depth interviews, the story and understanding of documents Involving those who are considered key players in providing education in the regular classroom for children with disabilities: children, parents or guardians, teachers and directors of institutions. This information was systematized through content analysis of categorical type, encoding and cast in matrices. As results of the development of this research, the meanings were identified from three subcategories: canonical, exceptional and negotiations with respect to disability, school integration and inclusion.

Keywords: education, disability, integration, inclusion, meanings, actions.

\section{Presentación}

El término educación, como se conoce en la actualidad, se ha constituido en la fuente de las expectativas y proyecciones acerca del desarrollo social en el marco del fenómeno de la globalización. De esta manera, se empieza a hablar acerca de la sociedad del conocimiento y de cómo ésta se constituye en el vehículo de la transformación social a través de las herramientas que hacen posible el acceso masivo a la información e indirectamente a la formación.

En ese sentido, se empiezan a difundir términos como inclusión, integración, multiculturalismo y diversidad en espacios formales e informales de educación. Se constituye la ciudad educadora y los individuos como ciudadanos del mundo con acceso a la información y participación, lo cual trae consigo reformas en la ley y nuevas concepciones acerca del umbral de educabilidad otrora establecido por pruebas de coeficiente intelectual y reglamentos estudiantiles.

La educación en Colombia como fuente de oportunidades, empieza a abrir sus puertas a la diversidad, se establecen leyes, decretos y normas técnicas que plantean las consideraciones mínimas para la atención educativa a este tipo de población.

Por tanto, se encuentra un escenario que legalmente está preparado para la atención educativa a personas en situación de discapacidad. Aún así, en el transcurso de estos 15 años de elaboración del soporte para esta atención, las instituciones de educación se han encontrado con inconvenientes en este proceso.

Gracias a estudios realizados por el DANE y la OMS (citados en Ospina, 2006), se encuentran datos que hablan acerca de la cobertura y deserción de la educación de personas en situación de discapacidad, lo cual evidencia que el esfuerzo por la inclusión va más allá de la asignación de cupos para este tipo de población o las modificaciones físicas de las instituciones educativas, pues tal como lo plantea Echeita (1994):

El problema no es la integración escolar en sí misma. El problema somos nosotros, nuestros propios límites conceptuales, nuestra capacidad para diseñar un mundo diferente, un sistema escolar no homogéneo, en el que cada cual pudiera progresar junto con otros, en función de sus necesidades particulares, y que pueda adaptarse para satisfacer las necesidades educativas de cada alumno, de la mano del profesorado que acepta y está preparado para enfrentarse a la diversidad (Echeita 1994, citando en Arnaíz, 1997, p. 313).

De esta forma, se evidencia la importancia de comprender los elementos que implican la atención educativa de calidad a niñas y niños en si- 
tuación de discapacidad en aulas regulares, en la cual, los significados de los protagonistas adquieren relevancia, en la medida en que como lo afirma Bruner (2002) hacen parte de las diversas construcciones de la realidad y abordan contextos amplios en los que no es suficiente recoger lo que las personas hacen, sino también, sus creencias, deseos, intenciones y compromisos, con el objetivo de negociar diferencias y lograr nuevas elaboraciones.

\section{Marco conceptual}

La atención educativa en el aula regular a niños y niñas en situación de discapacidad, puede ser comprendida a partir de los elementos que la agencian, tales como discapacidad, integración e inclusión escolar. De esta manera, se encuentran acepciones de discapacidad desde dos enfoques particulares; el primero de ellos, se puede evidenciar en una definición aportada por la OMS (1981) citada en Ospina (2004): "una discapacidad es toda restricción o ausencia (debida a una deficiencia) de la capacidad de realizar una actividad en la forma o dentro del margen que se considera normal para un ser humano" (p. 94).

Esta posición, hace referencia a una concepción de discapacidad como condición del individuo y como la ausencia de, que impide que éste pueda accionar en su entorno como lo hacen los otros.

Por otro lado, se empiezan a encontrar nociones de discapacidad desde un enfoque que complejiza la comprensión del fenómeno centrando su interés en el contexto más que en el sujeto, como se observa en la definición aportada por la Política Pública de Discapacidad para Bogotá Distrito Capital, Decreto № 470 (2007):

La discapacidad se entiende como el resultado de una relación dinámica de la persona con los entornos políticos, sociales, económicos, ambientales y culturales donde encuentra limitaciones o barreras para su desempeño y participación en las actividades de la vida diaria en estos entornos" (p. 18).
En ese sentido, la discapacidad se concibe como condición del entorno y no del individuo únicamente.

De acuerdo con esto, se puede evidenciar cómo a partir del cambio en las concepciones acerca de la discapacidad, se empiezan a gestar nuevas formas de comprenderla bajo características como la posibilidad de modificación de condiciones medio ambientales que permitan acceder al individuo a servicios y escenarios antes negados, y la oportunidad de participación en espacios escolares, laborales y lúdicos.

De esta manera, se encuentran también diferentes formas de concebir y abordar la atención educativa a niñas y niños en situación de discapacidad:

Mientras que en la integración escolar el énfasis está en la adaptación de la enseñanza en función de las necesidades específicas de los niños integrados, en la inclusión el centro de atención es la transformación de la organización y la respuesta educativa de la escuela para que acoja a todos los niños y tengan éxito en su aprendizaje (Blanco, 1999, p. 57).

Esta postura, evidencia una atención educativa diferente de acuerdo con la concepción de integración o inclusión, en ese sentido, la primera se relaciona de manera más estrecha con la definición de discapacidad como condición del individuo, mientras que la segunda, tiene que ver con la participación y modificación de contextos.

La concepción de inclusión parece ir más allá de la adecuación de espacios físicos y la atención especial a personas en situación de discapacidad, y según Hernández (2008) debe ir ligada a procesos de atención educativa que redefinan significados en torno a la discapacidad y a su manejo en la educación regular.

De esta forma, se observa una estrecha relación entre los significados y los contextos en los que estos se construyen, y se evidencia cómo la comprensión de esta relación es de vital importancia para la construcción de interpretaciones complejas, pues tal como lo propone Bruner (2002): “Sólo 
podremos comprender los principios que rigen la interpretación y elaboración de significados, en la medida en que seamos capaces de especificar la estructura y coherencia de los contextos más amplios en que se crean y transmiten significados específicos" (p. 73).

Por tanto, en la investigación se realizó una revisión de los significados en las narraciones de los protagonistas, en los cuales, se evidenciaron dos elementos fundamentales que cumplen una función mediadora; lo canónico y lo excepcional: "los significados entran a mediar lo culturalmente establecido (lo canónico) con lo inusual (lo excepcional)" (Arcila, Mendoza, Jaramillo \& Cañón, 2009, p. 43). En consecuencia, explorar en las narraciones aquello que es aceptado y aquello que se sale de la norma con respecto a la discapacidad y a su atención educativa en el aula regular, se constituye en una pista para comprender las negociaciones que orientan su gestión en contextos particulares.

\section{Método}

La investigación se realizó a partir de algunos principios de la etnometodología, entre los cuales de acuerdo con Galindo (1998), Guarné (2005) y Urbano (2007), se destaca el lenguaje como instrumento que organiza las experiencias de los sujetos, la comprensión del contexto para interpretar la realidad y el conocimiento social que es constantemente recreado en cada nuevo encuentro.

\section{Protagonistas de la investigación}

Los protagonistas de la investigación fueron tres niños y niñas en situación de discapacidad, 13 niños y niñas sin discapacidad, nueve padres de familia, cuatro profesores de grado quinto, dos profesores de grado sexto, dos rectores y dos coordinadores de tres colegios departamentales.

Los criterios de selección de las instituciones fueron tener por lo menos una niña o niño en situación de discapacidad (sensorial, motriz o cognitiva) en el aula regular en los grados quinto o sexto. De igual manera, se tuvo en cuenta la disponibi- lidad y accesibilidad tanto de la institución como la de los protagonistas. Para garantizar estas condiciones se contó con la asesoría de la Secretaría de Educación del municipio.

\section{Estrategias investigativas}

Entrevista a profundidad: se realizó con cada protagonista (niñas, niños, padres o acudientes, profesores, orientadores y directivas), y específicamente, con los padres o acudientes y las niñas y niños en situación de discapacidad se construyó un relato de un día como parte de la entrevista.

Construcción de cuentos: implicó la elaboración de cuentos realizados por las niñas y niños sin y en situación de discapacidad como parte de la entrevista.

Comprensión de documentos: implicó la revisión de documentos tales como el currículo, el PEl y el manual de convivencia de cada una de las instituciones.

\section{Etapas del proceso investigativo}

La Investigación se llevó a cabo a través del siguiente proceso:

1. Consolidación marco teórico acerca de los significados, discapacidad, integración e inclusión escolar.

2. Concreción de la estrategia metodológica, en la cual se establecieron la población y los instrumentos de investigación, realizando la validación de éstos a través de la revisión de expertos y pruebas piloto.

3. Desarrollo de las estrategias investigativas.

4. Sistematización y análisis de la información que se realizó a través del análisis de contenido de tipo categorial y la codificación y el vaciado en matrices, en las cuales se organizó la información obtenida a partir de las estrategias investigativas (entrevistas, construcción de cuentos y comprensión de documentos). Las categorías surgen del marco conceptual.

Posteriormente, se desarrolló un segundo nivel de análisis a partir de un ejercicio de tipo comprensivo acerca de los significados. 


\section{Resultados}

\section{Significados acerca de la discapacidad}

\section{Niños y niñas}

En esta categoría se evidencian significados de discapacidad por parte de los niños y niñas sin y en situación de discapacidad relacionadas con construcciones que parten de acuerdos establecidos con los adultos, tales como enfermedad, deficiencia física y déficit en determinadas asignaturas (español- matemáticas), las cuales son propias del sujeto. De esta manera, se observa en las narraciones de estos protagonistas negociaciones que se han desarrollado tanto en los contextos institucionales más cercanos (escolar y familiar) como en aquellos que hacen parte de su vida cotidiana (amigos, vecinos), es así como un niño expresa:

Niño(a): (La discapacidad es) a veces cuando las personas están enfermas y varias cosas

Inv1: ¿Hay alguna diferencia que de pronto ellos hagan que tu no hagas o al contrario que tú hagas algo que ellos no puedan hacer?

Niño(a): que ellos están en sillas de ruedas, uno corre y ellos no pueden

Inv1: ¿Conoces a alguien que esté en una situación así?

Niño(a): a Alonso, que jugamos y el no juega (niño quinto de primaria)

De esta manera, es posible identificar la correspondencia de estos significados con un modelo biomédico de la discapacidad, tal como el descrito por Amate (2008), en el cual se plantea una relación lineal entre etiología, patología y manifestaciones de las deficiencias. En ese sentido, no se consideran factores personales, respuestas emocionales, asuntos familiares o del contexto como componentes de la sintomatología.
En cuanto a los elementos de los significados, los niños y niñas sin discapacidad expresan de forma canónica que los niños en situación de discapacidad tienen menos capacidades y posibilidades que ellos, narrándose a sí mismos como "normales", lo cual comprenden desde las acciones desarrolladas en el aula, en tanto que a los niños y niñas en situación de discapacidad les asignan tareas más sencillas. De igual manera retoman expresiones que evidencian el trato que reciben sus compañeros en situación de discapacidad de parte de algunos pares: "la tratan mal, le dicen que es retrasada, que es como loca" (niña quinto de primaria).

Este aspecto se evidencia en los significados construidos por los niños y niñas en situación de discapacidad, quienes coinciden desde lo canónico al comprender la discapacidad como diferencias con respecto a su grupo de pares en el nivel de la ejecución y evaluación en las demandas académicas, al igual que las negociaciones establecidas por los adultos involucrados en sus contextos inmediatos: "mi hermana me dice que soy una bruta, que no sé ni siquiera sumar ni restar" (niña de quinto de primaria), lo cual se presenta como canónico en tanto que se convierte en parte de sus propias narraciones: "porque yo no soy inteligente, o sea, para el estudio.... El año pasado yo perdí el año o sea no me pude graduar" (niña quinto de primaria).

De esta manera, se puede observar cómo los contextos escolar y familiar desde sus demandas, establecen los criterios de normalidad y anormalidad que se constituyen en el eje central de las negociaciones sobre discapacidad en las instituciones. En consecuencia, se evidencia cómo los niños que no se encuentran en situación de discapacidad construyen significados de ésta como enfermedad e identifican a las personas que la poseen como especiales, compartiendo algunos criterios para establecer a quien se le dice especial y porque, invitando a su narración las conversaciones de los adultos y personas en situación de discapacidad diferentes al niño del curso:

Él es un niño especial, porque yo según lo que escuché en primero, es que él tenía la fiebre 
muy alta que se le pasó y se volvió así (niña quinto de primaria)

Con respecto a dichos criterios se identifica; la afectividad y el desempeño académico. En el primero, los niños y niñas expresan cómo las relaciones que establecen los niños y niñas en situación de discapacidad con sus pares se cargan de una afectividad diferente, lo cual se evidencia cuando los describen como más afectivos y cariñosos con sus compañeros; y el segundo; se presenta como una "enfermedad de la cabeza" que no les permite realizar las mismas tareas y actividades que los otros. Estos dos aspectos tienen según los niños y niñas una relación estrecha, ya que dicha "enfermedad de la cabeza" requiere tratos y relaciones especiales y diferentes.

De otro lado, se evidencia el aspecto excepcional cuando los niños y niñas expresan en sus narraciones que ser especial como discapacidad no significa estar limitado para aprender, lo cual empieza a ser parte de una negociación, en tanto que la presencia de niños y niñas en situación de discapacidad en el aula refleja un sentido particular, por lo que expresan que sus compañeros en situación de discapacidad si pueden aprender o por lo menos se esfuerzan para ello identificando sus diferencias en el tiempo de la ejecución de un ejercicio o la realización de tareas complejas.

De esta manera los niños y niñas comprenden la educación como derecho y expresan que a una persona en situación de discapacidad no se le puede discriminar, haciendo referencia a la posibilidad de acostumbrarse a las formas de relacionarse de los niños y niñas en situación de discapacidad y el respeto de su nivel cognitivo, físico y personal.

\section{Padres o acudientes}

Con respecto a los significados de discapacidad, en su aspecto canónico, los padres y acudientes afirman no tener conocimiento suficiente al respecto, ante lo cual se refleja el aspecto excepcional en tanto que deben explicar que ésta puede definirse como las limitaciones físicas de una persona y como la enfermedad mental en otros (refiriéndose a las discapacidades cogniti- vas), asumen y comprenden el término como una situación triste que limita a las personas a hacer lo que los sujetos normales si pueden, así mismo muestran sentimientos de comprensión y solidaridad, ya que consideran que es la falta de algo y que por eso no pueden desarrollarse normalmente en la vida social.

Al respecto, la excepcionalidad empieza a hacer parte de las negociaciones de los significados acerca de la discapacidad al retomar un concepto como la discriminación y la necesidad de abordar las limitaciones de los sujetos como responsabilidad colectiva, lo cual se refleja en la forma como manejan el tema con sus hijos en términos de solidaridad, tolerancia y comprensión, mostrándoles constantemente que los niños en situación de discapacidad tienen los mismos derechos que ellos, por tanto, estas construcciones se acercan a definiciones como la de Ospina (2006): "Complejo conjunto de condiciones, muchas de las cuales son creadas por el ambiente social. Su manejo requiere la actuación social y es responsabilidad colectiva de la sociedad" (Ospina, 2006, p. 111).

\section{Profesores}

Lo canónico, lo excepcional y las negociaciones.

Para desarrollar la categoría significados construidos por profesores con respecto a la discapacidad, se tuvo en cuenta su historia académica, en la cual se identifica la licenciatura en educación como formación básica y la ausencia de capacitación con respecto a la discapacidad. Aún así, gran parte de los profesores afirmaron tener experiencias en el trabajo con personas con discapacidad en situaciones de educación especial, por lo cual, se evidencia como excepcional la apertura del aula regular a esta población, generando sorpresa e inconformidad entre los profesores y directivas debido a la complejidad del tema y su preparación, especialmente por el tipo de discapacidad abordado, pues evidencian conocimientos acerca de la discapacidad física, mientras que la cognitiva plantea retos adicionales para los cuales afirman no han recibido capacitación.

En ese sentido, los profesores reclaman los apoyos necesarios para lograr el aprendizaje de los estudiantes con este tipo de discapacidad, debi- 
do al compromiso de las funciones cognitivas que consideran necesarias para el desarrollo de procesos en el aula regular.

Con respecto a las negociaciones, se evidencian significados con respecto a la discapacidad que han sido construidos a partir de su formación académica, historia personal y experiencia en la institución, en los cuales se encuentra que esta se define como dificultades tanto físicas como intelectuales que imposibilitan a las personas a desenvolverse en el medio como los otros.

...niños especiales, a todos se les llama niños especiales y no debería ser así... porque un niño especial, se considera que está sobrado... y no se debería, ni se le debería decir nada, ni tampoco que ellos se den cuenta de que son niños difer...ni tampoco me gusta niños diferentes, tampoco, y no sé qué término sería... (profesora quinto de primaria).

Se observa cómo los protagonistas presentan inconformidad frente a los términos producto de las negociaciones para referirse a los niños y niñas en situación de discapacidad, ya que no ven reflejados en estos sus comprensiones, por lo cual, se convierte en excepcional la construcción de términos que den cuenta de éstas. De igual manera, se observa que aunque se atribuye al medio una responsabilidad frente al desarrollo de los individuos en situación de discapacidad, se percibe como una condición del sujeto, quien debe aportar todos sus recursos para superar las dificultades propias de la deficiencia. Por lo tanto, se encuentra la referencia "niños con problemas" para identificar a esta población en la institución, y los acuerdos que se dan dentro del aula, entendiendo así que su manejo va dirigido a la comprensión, paciencia, tolerancia y colaboración.

Estas comprensiones son discutidas por Martínez (2005) cuando hace referencia a estas acepciones que al parecer buscan minimizar el impacto de la discapacidad en el aula regular y se convierten en nuevas formas de exclusión:

...aunque con nuevas palabras y justificaciones, estamos corriendo el riesgo de volver a los viejos tiempos de etiquetación y superespecialización que supuestamente queríamos superar. Dado que las mayorías poderosas y las clases dominantes que toman decisiones en educación siguen elevando a categoría científica ciertos prejuicios sobre las personas más vulnerables a las que eufemísticamente ahora denominan "especiales" o "diferentes". $Y$ que con frecuencia son construidos a través de los medios de comunicación y la cultura social vulgar, aunque, también, de ellos se hacen eco algunos docentes "desbordados". Quienes al no saber cómo tratarlos, comienzan a identificarlos de manera cada vez más temprana con las medidas a las que están deseando derivarlos (p.10).

En cuanto a políticas y leyes correspondientes al tema, los protagonistas afirman desconocer información concreta al respecto. Así mismo, en la institución no hay documentos que sustenten o den algún tipo de orientación al proceso de atención educativa de niños y niñas en situación de discapacidad dentro del aula regular.

\section{Directivas}

Con respecto a esta categoría se encuentran significados con respecto a la discapacidad que parten de las experiencias de los protagonistas en la institución y en el contexto familiar. De esta manera se evidencia que desde el aspecto canónico en la mayoría de directivos se encuentran comprensiones relacionadas con la limitación del sujeto para responder a las exigencias del medio, tal como lo hacen los niños y niñas sin discapacidad: "la discapacidad es la dificultad que presenta el ser humano para un desarrollo total en todas sus habilidades, comparado con los de las personas normales" (Coordinador).

Como aspecto excepcional, se empieza a reconocer el medio como factor que favorece las posibilidades de acción de los niños y niñas y a construir negociaciones con respecto a la discapacidad que se aparta de las concepciones tradicionales de normalidad y anormalidad: "la discapacidad es un estado diferente dentro de un medio, es un estado cognitivo, es un estado físico, es un estado 
emocional que es diferente en el medio el cuál se presenta" (Coordinadora).

Esta diversidad de comprensiones evidencia la dificultad para la construcción de una definición que responda a la experiencia:

Puesto que la experiencia de la discapacidad es única para cada individuo, no sólo porque la manifestación concreta de la enfermedad, desorden o lesión es única, sino porque esa condición de salud estará influida por una compleja combinación de factores...ello da pie para sugerir la imposibilidad de crear un lenguaje transcultural común para las tres dimensiones de la discapacidad (Egea \& Sarabia, 2001, p. 1).

\section{Significados acerca de la atención educativa en el aula regular}

\section{Niños y niñas}

En esta categoría se evidencian aspectos relacionados con la integración desde la postura de Hernández (2008), quien expone que su principal objetivo es la socialización, lo cual se presenta cuando los niños y niñas del salón se perciben como amigos de sus compañeros en situación de discapacidad por compartir el espacio físico y actividades dentro del aula. En ese sentido, los niños y niñas sin discapacidad han construido significados de la amistad desde criterios como cantidad de años compartidos y las relaciones de trabajo establecidas en el aula, tales como el préstamo de útiles o tareas.

De esta manera, la amistad en estos términos se establece como aspecto de la integración en tanto que los niños y niñas expresan que la mayoría de las veces sus compañeros en situación de discapacidad los buscan para jugar y dependiendo del juego pueden integrarse o no, de igual forma, se evidencia que son las niñas quienes comparten situaciones de juego con los niños y niñas en situación de discapacidad y ofrecen su compañía durante el descanso.
Por otro lado, se pueden evidenciar significados sobre integración cuando los niños expresan la intención de proteger a sus compañeros en situación de discapacidad de las agresiones de niños de otros cursos, ante lo cual expresan molestia al parecerles injusto que se comporten mal con un niño "enfermito". Estos significados surgen de las negociaciones establecidas en los contextos escolar y familiar, en los cuales se acuerda la importancia de la protección hacia las personas especiales.

Con respecto a estas negociaciones, los niños y niñas exponen la existencia de normas específicas establecidas por las profesoras sobre las acciones en el aula y las formas de relación con sus compañeros con discapacidad, entre las cuales se encuentra el no interrumpir sus actividades durante las clases, ya que esto puede generar dispersión del grupo y comportamientos agresivos que incluyen golpes de los niños sin discapacidad hacia sus compañeros en situación de discapacidad. De esta manera, las profesoras plantean trabajos en grupo con restricciones para la inclusión, asignando actividades en solitario que garanticen la estabilidad del aula.

Por otro lado, en cuanto a las actividades como aspecto de la integración, los niños y niñas expresan que éstas difieren cuando las asignan a sus compañeros en situación de discapacidad, lo cual hace referencia a esta categoría en tanto que su contenido según los niños y niñas no tiene que ver con las temáticas que se desarrollan como parte del plan de estudios. Entre las actividades asignadas a los niños y niñas en situación de discapacidad, se encuentran los dibujos y las planas. En ese sentido Lewis (2000) plantea que "La integración como sinónimo de colocación también pasa por alto el proceso de cambio de un sistema segregado a uno integrado" (Lewis, 2000, p. 16), por lo cual, el "estar en el aula" se constituye en la condición central de la integración obviando el proceso de aprendizaje que implica recursos humanos y físicos adicionales.

Este aspecto se ve reflejado en los significados del contexto escolar que evidencian los niños y niñas en situación de discapacidad, quienes en sus na- 
rraciones acerca del colegio presentan sentimientos de desagrado o aburrimiento expresando desarrollar actividades más satisfactorias en otros contextos como el hogar, lo cual se conecta con las narraciones de los niños y niñas sin discapacidad, quienes reconocen la soledad y la diferencia como los términos más comunes para referirse a sus compañeros con discapacidad.

Con respecto a la inclusión, se evidencia que la mayoría de niños y niñas sin discapacidad empiezan a construir significados sobre la atención educativa desde esta propuesta, lo cual se observa en expresiones que hacen referencia a la importancia de ayudar en el desarrollo de tareas a sus compañeros en situación de discapacidad con el fin de evitar el castigo de la profesora, y la posibilidad de tener un buen desempeño en las asignaturas siempre y cuando se les enseñe despacio y con mucho cuidado.

Como negociaciones que surgen de la excepcionalidad, se evidencia la inclusión de niños y niñas en situación de discapacidad cuando sus pares sin discapacidad hacen referencia a la importancia del acompañamiento y ayuda por parte de los profesores, la comunicación directa y el interés que estos demuestran en el trabajo desarrollado por los estudiantes, afirmando que estas son las cualidades de sus profesores favoritos y las cuales tendrán implicaciones en la ejecución adecuada de una tarea o la posibilidad de perder una asignatura.

Se logra identificar cómo en lo canónico los niños y niñas expresan que no existen diferencias en la didáctica de los profesores, afirmando que estos utilizan los mismos instrumentos y reglas para el trabajo en clase, las tareas y las obligaciones. En ese sentido, emerge una comprensión sobre la influencia que tiene el medio en el desarrollo académico de todos los niños y niñas sin y en situación de discapacidad, atribuyendo a factores como el hacinamiento y la desobedicencia de algunos compañeros, las dificultades en la concentración de los niños y niñas en situación de discapacidad quienes podrían requerir mayor atención.

En términos de lo excepcional los niños han logrado comprender las cualidades y potencialidades de los niños en situación de discapacidad cuando resaltan sus recursos dentro del salón: "cuando se atrasan no se quedan esperando y perdiendo el tiempo sino que siguen adelante y luego piden ayuda" (niña de quinto grado).

Por otro lado, se evidencian significados por parte de los niños y niñas en situación de discapacidad con respecto a la inclusión a través de sus expectativas de participación en la institución ya que éstas de dirigen a aprender y jugar. De igual manera reconocen la participación de sus compañeros y las valoraciones realizadas por sus profesores en este proceso de aprendizaje.

\section{Padres 0 acudientes}

Los significados por parte de padres y acudientes de los niños y niñas sin discapacidad, con respecto a la atención educativa de estudiantes en situación de discapacidad en el aula regular desde la integración o la inclusión, comienzan como una construcción desde lo excepcional, ya que estos protagonistas afirman no tener conocimiento sobre este proceso, por lo cual requieren explicaciones acerca del tema.

De esta manera, al establecer acuerdos con respecto a la definición de inclusión escolar que surge de los planteamientos teóricos, los padres y acudientes evidencian dificultades en este tipo de atención debido a la dinámica del aula, en la cual afirman, los profesores no tendrían la paciencia ni el interés de atrasarse por enseñarle a los niños y niñas en situación de discapacidad. Consideran que las dificultades más frecuentes del proceso de integración son los malos tratos de los otros niños, la discriminación y el no poder hacer lo que los demás sí.

Como aspecto excepcional, los protagonistas resaltan que los niños y niñas en situación de discapacidad pueden tener intereses similares frente a sus pares sin discapacidad, establecer relaciones de amistad y compartir experiencias como compañeros de clase, lo cual a largo plazo favorecería la construcción de redes que fortalezcan una educación sin discriminación. De esta manera, estos significados se acercan a las construcciones sobre inclusión: "inclusión educativa, donde 
el centro de atención no es las NEE, ni el sujeto individual, sino los entornos que rodean a la persona y que requieren ser adaptados para la accesibilidad a todo nivel" (Vicepresidencia de la República, 2008).

Se puede evidenciar que los significados sobre esta atención educativa se encuentran en construcción en tanto que los padres y acudientes en su mayoría muestran desinformación respecto al tema y desconocimiento sobre cómo es su manejo dentro del aula, cómo es la relación de sus hijos con los niños y niñas en situación de discapacidad y las políticas y leyes involucradas. De esta manera, hacen énfasis en la rapidez con que se ha desarrollado el proceso.

Para finalizar, se establecen negociaciones con respecto a la integración escolar en términos de reunión de niños y niñas sin y en situación de discapacidad con fines de socialización.

Con respeto al concepto de inclusión como fin de la atención educativa a niños y niñas en situación de discapacidad en el aula regular, se evidencia que algunos padres de niños y niñas sin discapacidad, luego de establecer acuerdos con respecto al término y su diferencia frente a la integración, consideran que los niños en situación de discapacidad se ven afectados por estos procesos, en tanto que no reciben una atención educativa especializada que implica un carácter personalizado y particular, de acuerdo con la discapacidad presentada, debido a las condiciones del sistema educativo, tales como la cantidad de niños y niñas por salón que obstaculiza una atención permanente por parte de los profesores, y la falta de información y formación en la comunidad académica.

En cuanto a los significados construidos por padres y acudientes de niños y niñas en situación de discapacidad, se evidencian construcciones que parten de su historia y las experiencias en la institución y en ese sentido, se observa que independientemente del término utilizado, los padres construyen el significado de una atención educativa con características particulares en las cuales se carga al contexto de la responsabilidad tanto de socialización como de aprendizaje:
... nosotros esperamos es que al niño lo traten con respeto, que le valoren el tiempo de él...y que el colegio asuma como tal la educación del niño, que reciba un niño con discapacidad y le haga un plan de trabajo y un seguimiento, pero más la institución que no un profesor (madre de familia quinto de primaria).

Al parecer, estos significados de la atención educativa a la discapacidad en el aula regular son más cercanos al concepto de educación inclusiva que surge desde las construcciones académicas:

Cuando hablamos de escuela inclusiva la propia expresión nos remite a "escuela", acentuando así la importancia de las condiciones y capacidades de la organización escolar que hagan posible la inclusión. Ésta no dirime únicamente en el aula, ni es el resultado de actuaciones de personas individuales que hacen cosas admirables pero en aislamiento; es el centro en su conjunto el que ha de articular respuestas coherentes y globales a los retos que representa la diversidad (González, 2008, p. 84).

\section{Profesores}

Con respecto a los significados construidos por los profesores en cuanto a la atención educativa en el aula regular de niños y niñas en situación de discapacidad, se evidencia una comprensión clara de los términos integración e inclusión y sus diferencias.

...la inclusión tiene que ver con involucrar a los niños y acompañar a los niños en situación de discapacidad en el aula regular. Ya no se hablaría de que los niños estén solo en un lado... distantes y aparte; con otros ejercicios diferentes a los de los niños regulares, sino ya incorporarlos al aula... (profesora quinto de primaria).

De esta manera, se registran las diferencias entre integración e inclusión, pues según los protagonistas, la primera se refiere al proceso por el cual los niños y niñas en situación de discapacidad están presentes dentro del aula y pueden participar en las actividades del grupo, por tanto la aten- 
ción se dirige a su proceso social y estrategias de interacción y no a sus necesidades educativas, mientras que en la inclusión se reconocen dichas necesidades y su atención dentro del aula regular.

Como negociaciones que han surgido con respecto a este tipo de atención, expresan la imposibilidad de cumplir con los requerimientos de ésta debido al número de niños y niñas por salón y a la falta de preparación y material de ayuda, lo cual se refleja en la falta de disposición y tiempo para el desarrollo de la inclusión. Así, los acuerdos que se dan dentro del aula se establecen en torno a la tolerancia, comprensión y el buen trato.

Con respecto a los significados construidos sobre la inclusión, los profesores reconocen las dificultades que presentan los niños y niñas gracias a sus limitantes físicos o cognitivos, aún así, centran los mayores impedimentos en las interacciones que se dan en las instituciones, en tanto que los profesores sin capacitación al respecto se han negado a participar en procesos de inclusión obstaculizando el desarrollo académico de los niños y niñas en situación de discapacidad. Por tanto, como acuerdos que surgen de esta excepcionalidad y teniendo como referente que el contexto debe adecuarse a las necesidades de niños y niñas en situación de discapacidad, se mencionan alternativas como capacitaciones, información, personal profesional de apoyo y acuerdos con los padres de familia.

En ese sentido como aspecto excepcional se considera que la atención en el aula regular de los niños y niñas en situación de discapacidad está dirigida al desarrollo de logros en el nivel educativo o cognitivo de cada niño y niña a largo plazo, aún así desde lo canónico reconocen que este proceso no va más allá del seguimiento y orientación de sus aspectos sociales.

Para finalizar, desde lo canónico, se afirma que la administración pública no ha brindado información al respecto y no se cuenta con los profesionales necesarios para la atención integral de estos estudiantes. De igual forma se expone el desconocimiento con respecto a documentos que apoyen el proceso de inclusión.

\section{Directivas}

Con respecto a los significados construidos acerca de la atención educativa de la discapacidad en el aula regular, se encuentran negociaciones establecidas entre la excepcionalidad (imposición de la integración-inclusión), y lo canónico (formas establecidas de educación regular) que generan negociaciones expuestas por los directivos en las que se hace énfasis en la dificultad para desarrollar procesos efectivos sin recursos humanos especializados, sin la capacitación y preparación suficiente y sin el apoyo de otras instancias como la familia y los organismos del Estado. De esta manera, se reconocen significados sobre la atención educativa desde el enfoque de la integración.

Por otro lado, se empieza a evidenciar la comprensión de la atención educativa desde el enfoque de la inclusión a partir de procesos de gestión y desarrollo de convenios y proyectos como esfuerzo particular de las instituciones, evidenciando falencias en el apoyo del Estado: "Por otra parte, el colegio también tiene ayudas, generalmente interdisciplinarias, en lo oficial me ha parecido mucho más difícil, porque creo que en lo oficial no está dado..." (Rectora).

De esta manera, se evidencian carencias en los procesos de formación para fortalecer la inclusión, ya que tal como lo plantean Moriña \& Parrilla (2006), la ausencia de evaluación de las propuestas formativas y la participación de los integrantes de las instituciones en este ejercicio obstaculiza la reflexión y la continuidad de esfuerzos dirigidos a esta.

\section{Documentos institucionales}

Dentro de los documentos oficiales de las instituciones no se explicitan significados sobre la discapacidad, la integración o la inclusión escolar.

\section{Conclusiones}

Se concluye que; se identificaron los significados desde tres subcategorías: canónico, excepcional y negociaciones con respecto a la discapacidad, a la integración y a la inclusión escolar por parte de niños y niñas sin y en situación de discapa- 
cidad, padres y acudientes y directivas de tres instituciones educativas. Se destacan comprensiones desde la postura médica de discapacidad (normalidad - anormalidad) y desde el enfoque de integración acerca de su atención educativa en el aula regular por parte de profesores y padres de niños y niñas sin discapacidad.

De igual manera, se evidencian comprensiones desde el enfoque de la inclusión por parte de directivas y padres de niños y niñas en situación de discapacidad, quienes reportan la dificultad para su gestión. De otro lado, los niños y niñas se encuentran construyendo significados que parten de las negociaciones con los adultos (integración) y con sus compañeros en situación de discapacidad (inclusión).

Se concluye que los significados sobre discapacidad, integración e inclusión escolar se construyen y movilizan a partir de las experiencias de cada uno de los protagonistas en los contextos en los cuales han participado, de esta manera, se evidencian las negociaciones constituidas por los padres de niños y niñas en situación de discapacidad, quienes desde su cercanía con la discapacidad se aproximan a las comprensiones de ésta, condicionada por el medio más que como condición del individuo, y en ese sentido los significados de la atención educativa se piensan desde la inclusión, en tanto que conciben como posible la atención de la discapacidad en el aula regular con fines de aprendizaje y socialización siempre y cuando el medio lo posibilite.

En las directivas, se evidencia la construcción de significados sobre discapacidad condicionada por el medio, aún así, reconocen el predominio e implicaciones de comprensiones desde el modelo médico en la comunidad académica y por tanto la atención educativa a partir del enfoque de la integración.

En cuanto a los profesores y padres de niños y niñas sin discapacidad, se observan comprensiones de la discapacidad como condición del individuo y por ello la dificultad para su atención educativa desde el enfoque de la inclusión.
Por otro lado, se presentan negociaciones entre los niños y niñas sin y con discapacidad en el aula regular que empiezan a acercarse al enfoque de la inclusión, lo cual, parece dar pistas acerca de las formas de movilizar la atención educativa desde la participación de todos los protagonistas y por tanto desarrollar acciones coherentes con las posibilidades institucionales más allá de las exigencias estatales sobre este tipo de procesos.

Finalmente, se recogen los significados que parten de las negociaciones que cada institución hace con respecto a las directrices estatales acerca de la atención educativa de la discapacidad que tienen como propósito restringir la educación especial, y en ese sentido a partir de los resultados de esta investigación, se propone la discusión y futuros estudios acerca de la educación inclusiva, pues se considera que esta puede admitir diversas posibilidades para su gestión entre las que se pueden revisar ciertas formas de educación especial y educación regular, pues tal como lo plantea Barton, 1998, citado en Sandoval, López, Miquel, Durán, Giné \& Echeita (2002): “(La) educación inclusiva es participación de todos los niños y jóvenes y remover, para conseguirlo, todas las prácticas excluyentes" (p. 230).

\section{Referencias}

Amate, A. (2008). Discapacidad: lo que todos debemos saber. Organización Panamericana de la Salud. International NGO Publishers and EC Organizations.

Arcila, P., Mendoza, Y., Jaramillo, J. \& Cañón, O. (2010). Comprensión del significado desde Vygotsky, Bruner y Gergen. Revista Diversitas: Perspectivas en Psicología, 6(1), 37-49.

Arnaíz, P. (1997). Integración, segregación, inclusión. Murcia, España: 10 años de integración España. Servicio de publicaciones de la Universidad.

Blanco, R. (1999). Hacia una escuela para todos y con todos. Boletín Proyecto Principal de Educación, 48. 
Bruner, J. (2002). Actos de significado: más allá de la revolución cognitiva. Madrid: Alianza.

Decreto No. 470 del 12 de octubre de 2007. Política Pública de Discapacidad para el Distrito Capital. Alcaldía Mayor de Bogotá. Sistema de Discapacidad.

Egea, C. \& Sarabia, S. (2001). Clasificaciones de la OMS sobre discapacidad. Boletín del Real Patronato sobre Discapacidad, 50. Extraído de http://usuarios.discapnet.es/disweb2000/ art/ClasificacionesOMSDiscapacidad.pdf

Galindo, L. (1998). Técnicas de investigación en sociedad, cultura y comunicación. México: Addison Wesley Longman.

González, M. (2008). Diversidad e inclusión educativa: Algunas reflexiones sobre el liderazgo en el centro escolar. Revista Electrónica Iberoamericana sobre Calidad, Eficacia y Cambio en Educación, 6(2), 82-99.

Guarné, B. (2005). Tecnologías sociales de la comunicación. Barcelona: UOC.

Hernández, C. (2008, noviembre). Ponencia Integración e Inclusión Educativa. 1er Foro de Educación y Discapacidad, localidad de Bosa, Bogotá, Colombia.

Lewis, A. (2000). Convivencia infantil y discapacidad. México: Trillas.
Martínez, B. (2005). Las medidas de respuesta a la diversidad: posibilidades y límites para la inclusión escolar y social. Revista de Currículum y Formación de Profesorado, 9(1), 1-30.

Moriña, A. \& Parrilla, A. (2006). Criterios para la formación permanente del profesorado en el marco de la educación inclusiva. Revista de Educación, 339, 517-539.

Ospina, J. (2006). Políticas públicas y discapacidad una mirada reflexiva. Trabajo de grado no publicado, Universidad Santo Tomás, Bogotá, D.C., Colombia.

Sandoval, M., López, M.L., Miquel, E., Durán, D., Giné, C., Echeita, G. (2002). Index for inclusión. Una guía para la evaluación y mejora de la educación inclusiva. Revista Contextos Educativos, 5. 227-238.

Urbano, H. (2007). El enfoque etnometodológico en la investigación científica. Revista de Psicología, 13, 89-91. Lima Perú. Liberabit.

Vicepresidencia de la República. (2006). Lineamientos en integración educativa. Extraído el 10 de noviembre de 2008 del sitio Web de la Vicepresidencia de la República: http:// www.discapacidad.gov.co/p_publica/lineamientos-educacion.htm 